\title{
The First Case of Longitudinal Extensive Transverse Myelitis (LETM) Following the First Dose Vaccination with Sinopharm Vaccine
}

Sara Bagherieh ${ }^{1}$, Narges Ebraimi ${ }^{1}$, Omid Mirmosayyeb ${ }^{1}$, Mahdi Barzegar ${ }^{1}$, and Vahid Shaygannejad ${ }^{1}$

${ }^{1}$ Isfahan University of Medical Sciences

January 27, 2022

\begin{abstract}
Longitudinal Extensive Transverse Myelitis (LETM) is a recognized devastating post-vaccination complication. Global reports on post-vaccination side effects have reported cases of LETM following COVID-19 vaccination with BioNTech vaccine and AstraZeneca vaccines. Here, we report the first case of a patient who experienced LETM following the first dose of Sinopharm vaccine.
\end{abstract}

\section{TITLE PAGE}

The First Case of Longitudinal Extensive Transverse Myelitis (LETM) Following the First Dose Vaccination with Sinopharm Vaccine

Running title: A case of LETM following Sinopharm vaccination

Authors' list:

Sara Bagherieh ${ }^{\mathbf{1}}$ (sarabagherieh20@gmail.com)

Narges Ebrahimi ${ }^{\mathbf{1}}$ (nargesebrahimiy@gmail.com)

Omid Mirmosayyeb ${ }^{\mathbf{1 , 2}}$ (omid.mirmosayyeb@gmail.com)

Mahdi Barzegar ${ }^{1,2}$ (barzegar_mahdi73@yahoo.com)

Vahid Shaygannejad ${ }^{\mathbf{1}, 2}$ (v.shaygannejad@gmail.com)

\section{Affiliations:}

${ }^{1}$ Isfahan Neurosciences Research Center, Isfahan University of Medical Sciences, Isfahan, Iran.

${ }^{2}$ Department of Neurology, School of Medicine, Isfahan University of Medical Sciences, Isfahan, Iran.

\section{Corresponding author:}

Vahid Shaygannejad, MD, Prof. of Neurology

Isfahan University of Medical Sciences

Isfahan, Iran.

Mail:barzegar_mahdi73@yahoo.com 
Cell: +989133133550

Written informed consent was obtained from the patient to publish this report in accordance with the journal's patient consent policy.

WE declare none of the authors listed on the manuscript are employed by a government agency that has a primary function other than research and/or education. We also declare none of the authors submitting this manuscript is an official representative or on behalf of the government.

Authors' contributions:

Conceptualization: OM, MB, VSh.

Data curation: not applicable

Formal Analysis: not applicable

Funding acquisition: not applicable

Investigation: SB, NE.

Methodology: not applicable

Project administration: OM, MB, VSh.

Supervision: VSh.

Validation: not applicable

Visualization: not applicable

Writing - original draft: SB.

Writing - review \& editing: SB, NE, OM, MB.

The First Case of Longitudinal Extensive Transverse Myelitis (LETM) Following the First Dose Vaccination with Sinopharm Vaccine

\section{Abstract:}

Longitudinal Extensive Transverse Myelitis (LETM) is a recognized devastating post-vaccination complication. Global reports on post-vaccination side effects have reported cases of LETM following COVID-19 vaccination with BioNTech vaccine and AstraZeneca vaccines. Here, we report the first case of a patient who experienced LETM following the first dose of Sinopharm vaccine.

Keywords: COVID-19, Vaccine Side-Effect, Longitudinal Extensive Transverse Myelitis

\section{Introduction:}

Longitudinal Extensive Transverse Myelitis (LETM) is a subtype of transverse myelitis (TM) which extends along three or more consequent vertebral segments. Although a rare phenomenon, it is a recognized devastating post-vaccination complication.[1] Various possible etiologies are proposed for the onset of LETM. It can occur as a part of multiple sclerosis (MS) disease, neuromyelitis optica spectrum disorder (NMOSD), many other autoimmune diseases, or autoimmune inflammation due to infection or environmental factors trigger the exaggerated response of the immune system.[2] One of the most disputable environmental factors that is thought to be able to trigger the onset of LETM is vaccination. Despite its rare incidence, the disabling nature of the condition calls for immediate diagnosis and treatment.[3] Here, we report the first case of LETM following the second dose vaccination with the Sinopharm vaccine.

\section{Case presentation:}

A 68 years old female was admitted to Kashani hospital due to progressive numbness in her left foot. She had a history of a neuroinflammation attack 8 years ago, for which she was prescribed with a 3 -day course 
of corticosteroid, propranolol and clonazepam. There were no reported dysfunctions regarding her attention span and memory, her underlying conditions included diabetes, hypertension, hyperlipidemia. She had no family history of MS or any other neuroinflammatory condition of any sort.

After receiving the first dose of Sinopharm vaccine, she experienced no side-effects, not even pain or tenderness of the injection-site. Nevertheless, 3 days after the first shot, her right foot became numb but resolved on its own in 2 days. However, in the following week her left foot went completely numb and she complained about tingling along the left lower extremity. (Figure 1) Subsequently, the left side of her body was numb in days, leading to inability to stand, keep balance, and walk without aid. A blood test was done and her OCB and anti-MOG came back negative, her IgG index and FBS were normal, and a mild lymphocytic pleocytosis was present. In T1 weighted MRI section, no enhancement was observed. Vasculitis-specific tests all returned negative, except for NMO-Ab. 43 days after the first does, the diagnosis of LETM was made. (Figure 2)

She was put on corticosteroids as a 5-day course of 1000mg of methylprednisolone pulse therapy accompanied by 10 sessions of physiotherapy. Her symptoms resolved and she is now able to stand up straight, walk without aid, and keep her balance.

\section{Discussion:}

Transverse myelitis is a rare condition which has been associated with a number of viruses and vaccines around the world. The SARS-CoV2 virus is no exception. From the early days of the pandemic, there has been a growing body of evidence on post-COVID infection cases of TM.[4] Although virus-mediated LETM is indicated by current data to happen due to the immune systems hyperinflammatory response, rather than the virus itself, the presence of temporal relations with vaccination further empowers the hypothesis that LETM can in fact be a possible post-vaccination neurological side effect. [1, 5]

Trial data of the AstraZeneca (AZD1222) has reported a case of TM during the pre-approval stage with the condition developing 2 weeks after the second shot. Local and national observations on post-vaccination side effects have also reported cases of TM following vaccination with BioNTech vaccine in UK, USA, and Germany.[6] Nevertheless, the clinical data on Sinopharm vaccine on the safety and efficacy of the vaccines reported no cases of LETM following vaccination. The present case; however, demonstrate a strong temporal link to COVID-19 Sinopharm vaccination as symptoms occurred 3 days following the first dose, and a range of differential diagnosis candidates, including MS, , neurosarcoidosis, and COVID-19 infection, were excluded.[7, 8] MS was ruled out due to the absence of serological or radiological signs of demyelinated lesions, and the absence of confirmatory CT-PET findings argued against a neurosarcoidosis diagnosis. [9, 10]

This case highlights the importance of a comprehensive post-vaccination follow up in an attempt to achieve a more realistic picture of the adverse events resulting from the application of novel vaccines particularly given their rapid development in times of the pandemic.

\section{References:}

1. Pagenkopf C, Südmeyer M. A case of longitudinally extensive transverse myelitis following vaccination against Covid-19. J Neuroimmunol. 2021;358. doi:10.1016/j.jneuroim.2021.577606.

2. Ahamed F, Ganesan S, James A, Zaher WA. Understanding perception and acceptance of Sinopharm vaccine and vaccination against COVID-19 in the UAE. BMC Public Health. 2021;21:1602.

3. Al Khames Aga QA, Alkhaffaf WH, Hatem TH, Nassir KF, Batineh Y, Dahham AT, et al. Safety of COVID-19 vaccines. J Med Virol. 2021;93:6588-94.

4. Omeish H, Najadat A, Al-Azzam S, Tarabin N, Abu Hameed A, Al-Gallab N, et al. Reported COVID19 vaccines side effects among Jordanian population: a cross sectional study. Hum Vaccin Immunother. $2021 ;: 1-8$. 
5. Tan WY, Yusof Khan AHK, Mohd Yaakob MN, Abdul Rashid AM, Loh WC, Baharin J, et al. Longitudinal extensive transverse myelitis following ChAdOx1 nCOV-19 vaccine: a case report. BMC Neurol. 2021;21:395.

6. Gao J-J, Tseng H-P, Lin C-L, Shiu J-S, Lee M-H, Liu C-H. Acute Transverse Myelitis Following COVID-19 Vaccination. Vaccines. 2021;9.

7. Mallapaty S, Ledford H. COVID-vaccine results are on the way - and scientists' concerns are growing. Nature. 2020;586:16-7.

8. Mirmosayyeb O, Bagherieh S, Shaygannejad V. Acute CNS demyelination in a subject with cerebellar ataxia following the first dose of COVID-19 vaccine; a case report. Hum Vaccin Immunother. 2021;:1-3.

9. Remmel A. "It's a minefield": COVID vaccine safety poses unique communication challenge. Nature. 2021;593:488-9.

10. Davis CJ, Golding M, McKay R. Efficacy information influences intention to take COVID-19 vaccine. Br J Health Psychol. 2021.

Figure legends:

Figure 1 Sagittal T2 weighted image of thoracic spine (T8 - T12) shows hyperintense signal with wide axial and longitudinal extent.

Figure Axial T2 weighted section shows the involvement of over $50 \%$ of the spinal cord's central gray matter and white matter.

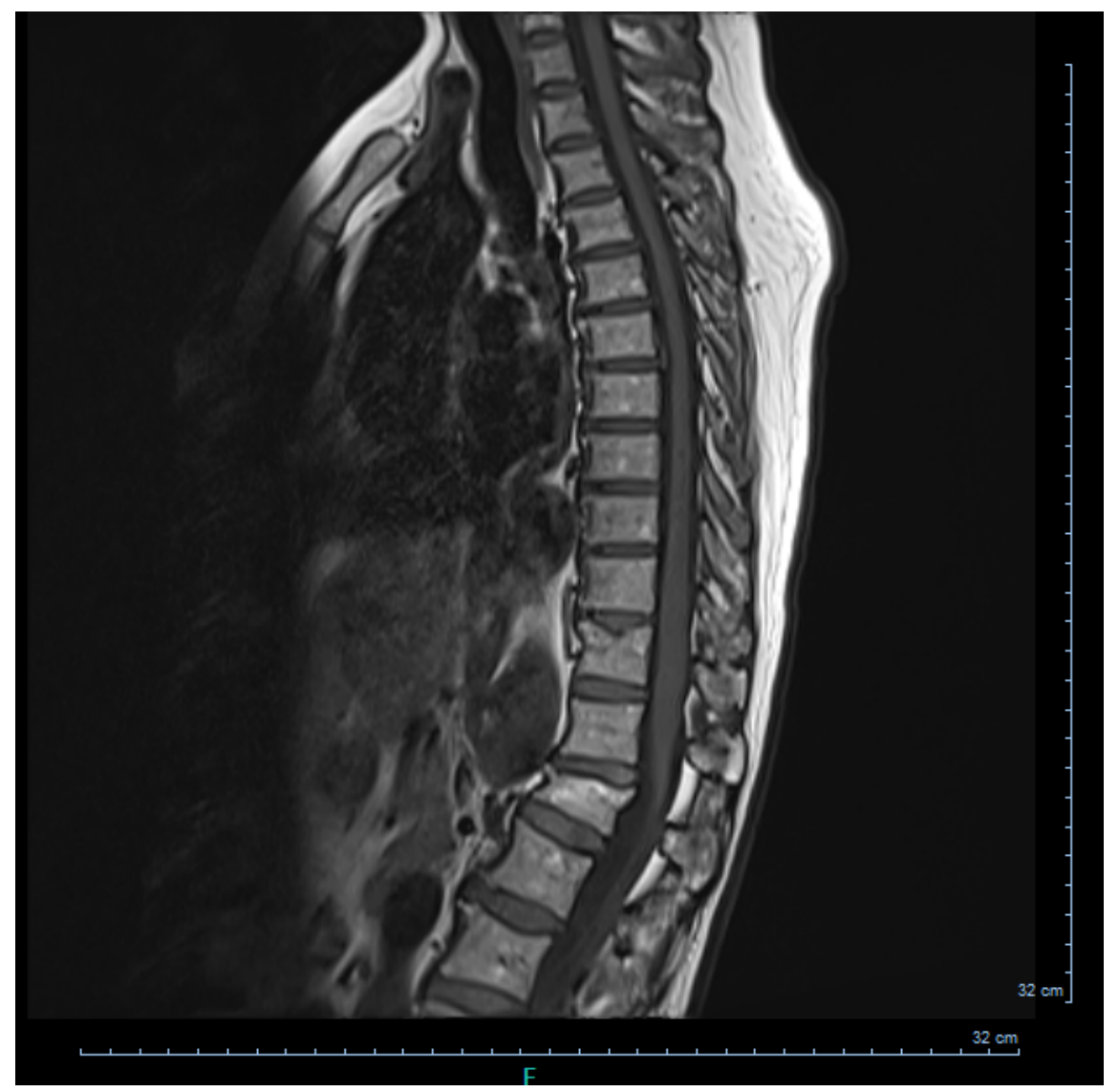




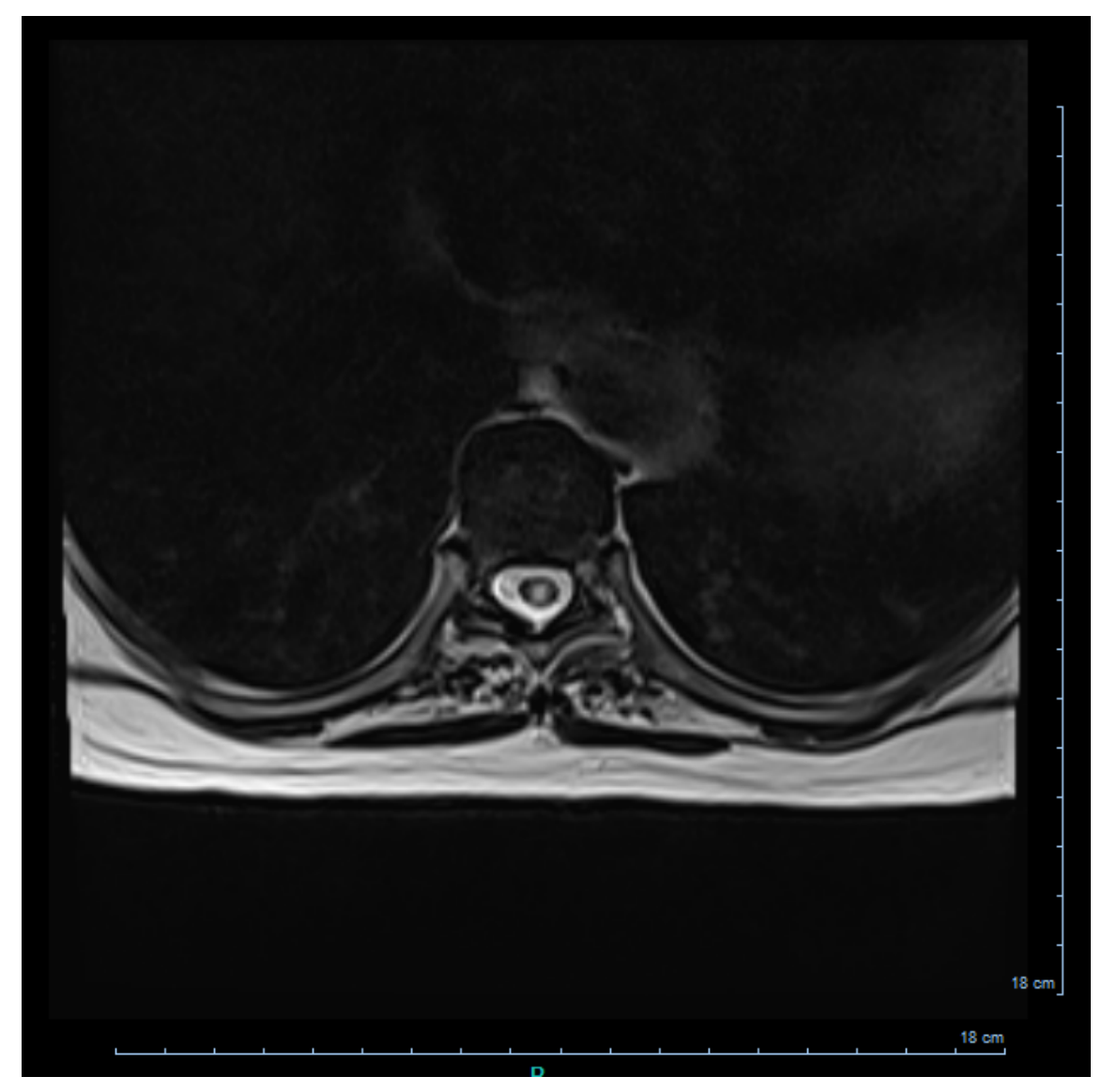

